Revue des sciences de l'éducation

Les manuels scolaires : réformes curriculaires, développement professionnel et apprentissages des élèves

Textbooks: curriculum reforms, professional development and student learning

Los libros de texto: reformas curriculares, desarrollo profesional y aprendizaje de los alumnos

Johanne Lebrun et Daniel Niclot

Volume 35, numéro 2, 2009

Les manuels scolaires : réformes curriculaires, développement

professionnel et apprentissages des élèves

URI : https://id.erudit.org/iderudit/038726ar

DOI : https://doi.org/10.7202/038726ar

Aller au sommaire du numéro

Éditeur(s)

Revue des sciences de l'éducation

ISSN

0318-479X (imprimé)

1705-0065 (numérique)

Découvrir la revue

Citer ce document

Lebrun, J. \& Niclot, D. (2009). Les manuels scolaires : réformes curriculaires, développement professionnel et apprentissages des élèves. Revue des sciences de l'éducation, 35(2), 7-14. https://doi.org/10.7202/038726ar 


\section{Les manuels scolaires: réformes curriculaires, développement professionnel et apprentissages des élèves}

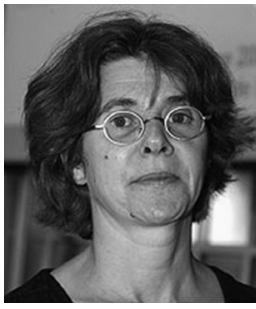

Johanne Lebrun, professeure Université de Sherbrooke

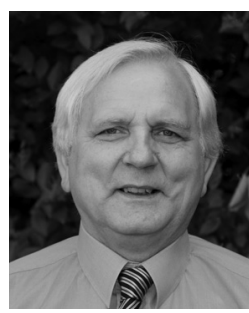

Daniel Niclot, maître de conférences Université de Reims Champagne-Ardenne

Quel que soit le niveau de classe ou la discipline concernée, les manuels scolaires ne sont pas uniquement destinés à un public d'enfants ou d'adolescents, comme leur dénomination de manuels d'élèves semblerait l'indiquer. Leur fonction est beaucoup plus globale. Ils occupent une position d'interface au sein du système éducatif, notion qui implique à la fois un contact et des échanges croisés avec leur environnement. Les travaux menés ces dernières années permettent d'identifier quatre points principaux de jonction entre les manuels scolaires et différents éléments du système éducatif et, plus largement, la société dans ses rapports avec l’École.

\section{Quatre points de jonction}

En premier lieu, les manuels scolaires se situent au carrefour de la demande sociale, de l'éducation et de l'édition. Les contenus pédagogiques et didactiques des manuels sont élaborés dans un contexte idéologique, politique, culturel et économique, dont l'influence ne doit pas être négligée (Niclot, 2003b; Niclot et Aroq, 2006). Produit socio-culturel, le manuel est également un produit commercial 
dans un certain nombre de pays (Choppin, 1992; Gérard et Roegiers, 1993; Stray, 1993). Plus précisément, les logiques économiques des maisons d'édition jouent un rôle important dans le choix des contenus, de la forme et de la présentation des manuels. Les stratégies économiques des entreprises d'édition scolaire peuvent également conditionner les innovations; par exemple, à l'heure actuelle en France par rapport aux médias éducatifs (Bahuaud, 2006).

Comme outil d'actualisation des prescriptions officielles, le manuel est, en second lieu, à la jonction du curriculum formel et du curriculum réel, c'est-à-dire entre les contenus prescrits et ceux qui sont réellement enseignés dans la classe. Souvent, les manuels se substituent aux programmes d'études (Julkunen, Selanders et Alhberg, 1991; Spallanzani, Biron, Larose, Lebrun, Lenoir, Masselter et Roy, 2001; Venezky, 1992; Woodward, Elliot et Nagel, 1988). En France plus particulièrement, l'accès aisé des enseignants aux manuels scolaires, grâce aux spécimens qui leur sont distribués gratuitement par les éditeurs, renforce encore cette tendance (Le Roux, 1997).

Le manuel assure, en troisième lieu, une position centrale entre l'enseignement et l'apprentissage. La recension de la documentation scientifique (Lebrun, Lenoir, Laforest, Larose, Roy, Spallanzani et Pearson, 2002) et les résultats de recherches récentes menées au Québec (Lebrun, 2001; Lenoir, 2002; Spallanzani et collab., 2001) témoignent de l'emprise des manuels scolaires sur les pratiques enseignantes et, par le fait même, sur les apprentissages des élèves. En France, les enquêtes menées depuis plus de 30 ans, dans l'enseignement secondaire notamment, vont dans le même sens (Citron et Marbeau 1972; Douzant-Rosenfeld, 1991; Tournier et Navarro, 1985). Ces études et ces enquêtes montrent que les dimensions curriculaire, pédagogique, didactique et évaluative des manuels contribuent en grande partie à définir les savoirs à enseigner, les stratégies pédagogiques et didactiques employées, la progression attendue des élèves, le cheminement qu'ils doivent parcourir pour acquérir les savoirs, leur degré de participation dans les activités et le mode de reconnaissance de leurs acquis. Les données des recherches québécoises rejoignent celles des quelques recherches en provenance de l'Amérique du Nord et de l'Europe occidentale. Selon des travaux américains et européens (Alverman, 1989; Armento, 1986; Gentil et Verdon, 1995; Hummel, 1988, Zahorik, 1991), le manuel scolaire détermine, pour une large part, les activités réalisées en classe. Il peut même apparaître, en France notamment, comme un medium participant aux évolutions de la professionnalité enseignante, en raison notamment des insuffisances de la formation continue (Niclot, 2003a).

Les savoirs, les méthodes et les activités développés par les auteurs dans les manuels ont une action le plus souvent directe sur les pratiques enseignantes, qui va bien au-delà d'une simple influence selon un terme couramment employé dans les écrits de recherche. Pour autant, les effets sur les pratiques sont divers et ne vont pas nécessairement dans le sens d'une meilleure adéquation entre les situations d'enseignement-apprentissage mises en œuvre et la réalité des classes et des élèves. 
Les enseignants peuvent commettre des contresens, des simplifications abusives, mal comprendre les méthodes ou les savoirs développés par les manuels, ou tout simplement faire preuve d'une trop grande soumission à l'égard des manuels et perdre leur autonomie professionnelle (Rey, 2001). Les savoirs et savoir-faire développés par les manuels sont aussi parfois en retrait par rapport aux prescriptions officielles (Clerc, 2001; Hasni et Roy, 2006; Lebrun, 2002). Selon les lieux, les contextes et les époques, les manuels peuvent représenter un facteur de stagnation ou contribuer à une transformation des types de situations d'enseignementapprentissage mises en œuvre par les enseignants dans leur classe, mais aussi de la manière dont les élèves apprennent.

La fonction de médiation entre les savoirs, les méthodes et les élèves, ce qui correspond à la fonction première des manuels, a paradoxalement été peu étudiée jusqu'à présent. En France par exemple, les manuels sont, dans la majorité des cas, conçus pour être utilisés de manière autonome par les élèves. Ils sont conçus comme un médium qui permet à l'élève d'avoir un accès direct aux savoirs et aux savoir-faire disciplinaires prescrits par les curricula. Plus généralement, le manuel contribue, par la nature des situations d'enseignement-apprentissage qu'il propose, à déterminer le parcours que l'élève devra suivre pour accéder au savoir. Ainsi, au-delà de l'organisation d'un corpus de savoir, c'est un certain rapport au savoir qui est pris en compte dans les manuels scolaires (Hasni et Roy, 2006; Lebrun, 2001, 2002, 2006; Lebrun, Lenoir et Desjardins, 2004 ; Lenoir, 2002 ; Lenoir, Rey, Roy et Lebrun, 2001 ; Spallanzani et collab., 2001).

Enfin, le manuel assure une quatrième fonction spécifique, en assurant la jonction entre le savoir savant et le savoir scolaire. Des études effectuées dans le cadre des didactiques des disciplines ont abordé cette question, à la lumière, notamment, des modèles expliquant le passage du savoir scientifique aux savoirs scolaires (Chervel, 1988; Chevallard, 1991). Plus récemment, des interrogations ont porté sur le traitement, par les manuels scolaires, des autres dimensions d'une discipline comme la chimie, qui est certes une science, mais aussi une technique et une activité industrielle (Terrien, 1998).

La position d'interface occupée par les manuels scolaires dont les aspects principaux viennent d'être explicités implique des interrelations avec le système scolaire.

\section{Contraintes extérieures}

Si les manuels ont une influence sur les pratiques des enseignants, les apprentissages des élèves et les processus de construction des savoirs scolaires, ils sont eux-mêmes dépendants de nombreuses contraintes extérieures. Ils doivent tenir compte des logiques de l'édition, des modèles disciplinaires, des pratiques ordinaires de classe qu'ils ne peuvent ignorer, sous peine, surtout dans les pays ou l'édition scolaire est le fait d'éditeurs privés soumis aux logiques économiques libérales, de perdre leur audience. Les éditeurs de manuels scolaires peuvent, en retour, influer directement 
sur les orientations générales des programmes. En France, un rapport de l'Inspection générale de l'Éducation nationale dénonce l'influence du groupe de pression constitué par les éditeurs de manuels scolaires, qui interviennent activement dans les discussions sur les réformes des programmes d'enseignement (Borne, 1998).

La question des interrelations multiples des manuels scolaires avec les différentes composantes du système scolaire apparaît donc centrale, dans le cadre des réformes curriculaires actuellement mises en œuvre dans de nombreux États du monde. Ces réformes se réfèrent à trois orientations théoriques principales fortement interreliées: le constructivisme, l'interactionnisme social et le relativisme épistémologique (Weil-Barais et Dumas Carré, 1998). Elles se sont traduites dans la plupart des cas par l'adoption d'une approche par compétences dans les curriculums d'enseignement. Si ces nouvelles perspectives curriculaires nécessitent une reconceptualisation de la pratique des enseignants, elles entraînent également celle des supports didactiques auxquels se réfèrent les enseignants, dont, en premier lieu, les manuels scolaires. Dans ce contexte, le rôle prépondérant du manuel scolaire dans l'intervention éducative soulève de nombreuses questions. L'évolution des manuels scolaires, leurs caractéristiques formelles, curriculaires, pédagogiques et didactiques sont-elles congruentes avec l'évolution des savoirs didactiques et disciplinaires? Avec les principes d'autonomie et d'expertise professionnelles? Une intervention éducative de type constructiviste peut-elle s'accommoder, voire s'appuyer, sur le cheminement proposé par le manuel scolaire? Existe-t-il une possible jonction entre la construction des savoirs par l'élève, la médiation exercée par l'enseignant et le manuel scolaire?

\section{Dans ce numéro}

Dans ce contexte, les articles proposés dans ce numéro qui a pour thématique les manuels scolaires: réformes curriculaires, développement professionnel et apprentissages des élèves traitent des fonctions et du rôle des manuels scolaires de l'enseignement primaire et secondaire caractérisés par les évolutions rapides des systèmes éducatifs.

Dans deux textes, on étudie d'abord les manuels scolaires au regard de leur rôle d'aide à l'actualisation des prescriptions officielles pour les enseignants.

Ainsi, Johanne Lebrun, professeure à l'Université de Sherbrooke, analyse certains manuels scolaires québécois pour l'enseignement de l'univers social au $3^{\mathrm{e}}$ cycle du primaire sous l'angle de l'incidence des changements curriculaires sur la configuration des démarches d'enseignement-apprentissage proposées. Le texte est ainsi centré sur la fonction d'actualisation des prescriptions officielles attribuée aux manuels scolaires, mais en prenant en considération, en amont, l'impact de la demande politico-éducative sur cette actualisation et, en aval, les conséquences potentielles de cette configuration sur le processus d'enseignementapprentissage.

Pour sa part, Suzanne-G. Chartrand, professeure à l'Université Laval, questionne l'adéquation de certains manuels de l'élève conçus pour l'apprentissage de l'histoire 
au $2^{\mathrm{e}}$ secondaire, du point de vue des compétences, pour la compréhension-interprétation en lecture que l'élève doit mobiliser. La fonction d'actualisation des prescriptions officielles des manuels scolaires est, cette fois-ci, analysée sous l'angle de la prise en considération du processus de lecture dans la structuration et la présentation du corpus disciplinaire en histoire.

Les manuels scolaires comme instruments de développement professionnel des enseignants est un thème plus particulièrement traité par trois textes qui font référence, l'un aux mathématiques et les deux autres, aux sciences.

C'est la question des types d'usages des manuels scolaires de mathématiques par les enseignants du primaire qui est abordée par Claire Margolinas (Université BlaisePascal Clermont-Ferrand) et Floriane Wozniak (Université Claude-Bernard Lyon 1). À partir d'entretiens semi-directifs réalisés auprès de onze professeurs expérimentés, sont analysés et décrits les types d'usage des manuels dans le cadre de l'enseignement des mathématiques à l'école élémentaire, et notamment pour la conception des leçons. Plus globalement, c'est une interrogation sur la fonction des manuels comme médium de formation des enseignants qui est menée dans cette étude.

De leur côté, Abdelkrim Hasni (Université de Sherbrooke), Ghislain Samson (Université du Québec à Trois-Rivières), Christine Moresoli (Université de Waterloo) et Marie-Ėve Owen (Université de Sherbrooke) présentent les résultats d'une enquête réalisée auprès d'enseignants québécois du premier cycle du secondaire, au regard de leurs attentes envers les manuels scolaires de sciences, dans le contexte d'implantation des nouveaux programmes d'études. C'est le rôle du manuel comme outil de formation et d'information pour les enseignants qui est au cour de la réflexion de cet article.

Quant à Elisabeth Plé (Université de Reims Champagne-Ardenne), elle pose la question qui s'apparente à un paradoxe de la contradiction qui existe entre les recommandations officielles de nature constructiviste figurant dans les programmes de sciences et l'utilisation des manuels scolaires comme supports de travail des élèves par les enseignants. Les ouvrages scolaires semblent en effet peu propices à des apprentissages qui s'appuient sur des démarches d'investigation empirique du monde. Dans cet article, l'auteur développe le concept de maître de papier, et explore les types de relations qui se nouent entre ces maitres muets que sont les manuels et les enseignants en chair et en os.

Enfin, les analyses de Daniel Niclot (Université de Reims Champagne-Ardenne) sont plus centrées sur les manuels eux-mêmes et sur leurs transformations à long terme. Les principales évolutions des manuels de géographie français de l'enseignement secondaire publiés depuis près de cent ans y sont mises en évidence. L'auteur identifie les différents modèles de manuels qui se sont succédé durant cette période. Les changements intervenus sont mis en relation avec l'évolution de la société françaises, de l'édition, de la demande institutionnelle, mais aussi avec celles du public scolaire, et notamment des types de rapports qu'entretiennent les élèves avec les savoirs scolaires. 
L’entrée privilégiée par ces textes est donc disciplinaire, puisque les articles proposés abordent le cas des manuels de sciences, de mathématiques et des sciences sociales (dont la géographie). Ils sont en outre ancrés dans des contextes nationaux correspondant à la France et au Québec. Pourtant, malgré la diversité des analyses réalisées et des référents théoriques mobilisés, tous ces textes, au-delà des exemples étudiés et des problématiques posées, se trouvent confrontés au caractère complexe et multidimensionnel des manuels scolaires. Les auteurs proposent des grilles de lecture et d'interprétation, des concepts heuristiques et généralisables qui contribuent, de toute évidence, à l'enrichissement du courant de recherche sur les manuels scolaires.

ENGLISH TITLE - Textbooks: curriculum reforms, professional development and student learning

TíTULO EN ESPAÑOL - LOS libros de texto : reformas curriculares, desarrollo profesional y aprendizaje de los alumnos

\section{Références}

Alverman, D. (1989). Teacher-student mediation of content area texts. Theory into practice, $27,142-147$.

Armento, B. J. (1986). Research on teaching social studies. Dans M. C. Wittrock (Dir.): Handbook of research on teaching ( $3^{\mathrm{e}}$ édition). New York, New York: Macmillan.

Bahuaud, M. (2006). Les éditeurs scolaires traditionnels à la recherche d'un modèle économique. Dans E. Bruillard (Dir.): Manuels scolaires, regards croisés. Caen, France: CRDP Basse-Normandie.

Borne, D. (1998). Le manuel scolaire. Programme de travail 1997-1998. Paris, France: ministère de l'Éducation nationale, de la Recherche et de la Technologie, Inspection générale de l'éducation.

Chevallard, Y. (1991). Transposition didactique - du savoir savant au savoir enseigné, Paris, France: La pensée sauvage.

Chervel, A. (1988). L'histoire des disciplines scolaires. Réflexions sur un domaine de recherche. Histoire de l'éducation, 38, 59-119.

Choppin, A. (1992). Les manuels scolaires: histoire et actualité. Paris, France: Hachette Éducation.

Citron, S. et Marbeau, L. (1972). Résultats d'une enquête effectuée en juin 1969-Instruments de travail en histoire et géographie dans l'enseignement secondaire: revues et manuels. Paris, France: Collection Recherches pédagogiques, Institut national de recherche pédagogique.

Clerc, P. (2001). Les manuels scolaires au risque de l'innovation. [En ligne]. Disponible le 22 mai 2008: http://xxi.ac-reims.fr/fig-st-die/actes/actes_2001/clerc/article.htm

Douzant-Rosenfeld, D. (1991). L'image de la géographie chez les professeurs et dans la population française. Bulletin intergéo, 104, 13-85. 
Gentil, R. et Verdon, R. (1995). Étude sur les outils pédagogiques à l'école primaire. Pratiques et opinions des enseignants. Les dossiers d'éducation et formations, $n^{\circ} 56$. Paris, France: ministère de l'Éducation nationale, de l'Enseignement supérieur, de la Recherche et de l'Insertion professionnelle, Direction de l'évaluation et de la Prospective.

Gérard, F.-M. et Roegiers, X. (1993). Concevoir et évaluer des manuels scolaires. Bruxelles, Belgique: De Boeck Université.

Hasni, A. et Roy, P. (2006). Comment les manuels proposent-ils d'aborder les concepts scientifiques avec les élèves? Cas des concepts de biologie. Dans J. Lebrun, J. Bédard, A. Hasni et V. Grenon (Dir.): Le matériel didactique et pédagogique: soutien à l'appropriation ou déterminant de l'intervention éducative. Québec, Québec: Presses de l'Université Laval.

Hummel, C. (1988). School textbooks and lifelong education: an analysis of schoolbooks from three countries. Hamburg, Allemagne: UNESCO Institute for Education.

Julkunen, M.-L., Selander, S. et Ahlberg, M. (1991). Research on texts at school. Rapport de recherche de la Faculté d'éducation $n^{\circ}$ 37. Joensuu, Finlande: Université de Joensuu.

Lebrun, J. (2001). Place et rôle du manuel scolaire en sciences humaines dans les planifications d'activités d'enseignement-apprentissage des futurs enseignants du primaire de l'Université de Sherbrooke. Dans Y. Lenoir, B. Rey, G.-R. Roy et J. Lebrun (Dir.): Le manuel scolaire et l'intervention éducative. Regards critiques sur ses apports et ses limites. Sherbrooke, Québec: Éditions du CRP.

Lebrun, J. (2002). Les modèles d'intervention éducative véhiculés par la documentation officielle et les manuels scolaires approuvés concernant l'enseignement-apprentissage des sciences humaines au troisième cycle du primaire. Thèse de doctorat inédite, Université de Sherbrooke, Sherbrooke.

Lebrun, J. (2006). Le manuel scolaire réformé: quelle place pour la médiation de l'enseignant et les apprentissages des élèves? Dans J. Lebrun, J. Bédard, A. Hasni et V. Grenon (Dir.) : Le matériel didactique et pédagogique: soutien à l'appropriation ou déterminant de l'intervention éducative. Québec, Québec: Presses de l’Université Laval.

Lebrun, J., Lenoir, Y., Laforest, M., Larose, F., Roy, G.-R., Spallanzani, C. et Pearson, M. (2002). Past and current trends in the analysis of textbooks in the Quebec context. Curriculum inquiry, 32(1), 51-83.

Lebrun, J., Lenoir, Y. et Desjardins, J. (2004). Le manuel scolaire « réformé» ou l'illusion du changement: analyse de l'évolution des critères d'évaluation des manuels scolaires de l'enseignement primaire entre 1979 et 2001. Revue des sciences de l'éducation, 30(3), 509-533.

Lenoir, Y. (2002). L'utilisation du matériel interdisciplinaire par les enseignants du primaire: impact sur leurs pratiques. Rapport de recherche déposé auprès du Conseil de recherches en sciences humaines du Canada (Recherche CRSH n 410-98-0307). Sherbrooke, Québec: Université de Sherbrooke, Centre de recherche sur l'intervention éducative (CRIE).

Lenoir, Y., Rey, B., Roy, G.-R. et Lebrun, J. (2001). Le manuel scolaire et l'intervention éducative. Regards critiques sur ses apports et ses limites. Sherbrooke, Québec: Éditions du CRP.

Le Roux, A. (1997). Didactique de la géographie. Caen, France: Presses universitaires de Caen. 
Niclot, D. (2003a). Et si les manuels scolaires étaient, par défaut, un outil de professionnalisation des enseignants? Dans G. Baillat, P. A. Martin et D. Niclot (Dir.): Vers quelle professionnalité enseignante en France et au Québec? Paris, France: Centre national de documentation pédagogique.

Niclot, D. (2003b). Les manuels de géographie de l'enseignement secondaire. En comprendre les logiques pour mieux les utiliser. Reims, France: Centre régional de documentation pédagogique.

Niclot, D. et Aroq, C. (2006). Les évolutions récentes des manuels de géographie de l'enseignement secondaire français et les pratiques déclarées des enseignants. Dans J. Lebrun, J. Bédard, A. Hasni et V. Grenon (Dir.) : Le matériel didactique et pédagogique: soutien à l'appropriation ou déterminant de l'intervention éducative. Québec, Québec: Presses de l'Université Laval.

Rey, B. (2001). Manuels scolaires et dispositifs didactiques. Dans Y. Lenoir, B. Rey, G.-R. Roy et J. Lebrun (Dir.) : Le manuel scolaire et l'intervention éducative. Sherbrooke, Québec: Éditions du CRP.

Spallanzani, C., Biron, D., Larose, F., Lebrun, J., Lenoir, Y., Masselter, G. et Roy, G.-R. (2001). Le rôle du manuel scolaire dans les pratiques enseignantes au primaire. Sherbrooke, Québec: Éditions du CRP.

Stray, C. (1993). Quia nominor leo: vers une sociologie historique du manuel. Histoire de l'éducation, 58, 71-102.

Terrien, M. (1998). Contribution à l'étude des échanges entre la recherche et l'enseignement supérieur (chimie). Thèse de doctorat inédite, Université d'Angers, Angers, France.

Tournier, M. et Navarro, M. (1985). Les professeurs et le manuel scolaire. Paris, France: Institut national de la recherche pédagogique.

Venezky, R.-L. (1992). Textbooks in school and society. Dans P. W. Jackson (Dir.) : Handbook of research on curriculum. A project of the American educational research association. New York, New York: Macmillan.

Weil-Barais, A. et Dumas Carré, A. (1998). Les interactions didactiques: tutelle et/ou médiation? Dans A. Dumas-Carré et A. Weil-Barais (Dir.): Tutelle et médiation dans l'éducation scientifique. Berne, Suisse: Peter Lang.

Woodward, A., Elliot, D. L. et Nagel, K. C. (1988). Textbooks in school and society. An annoted bibliography and guide to research. New York, New York: Garland.

Zahorik, J. A. (1991). Teaching style and texbooks. Teaching and teacher education, 7(2), 185-196.

\section{Correspondance}

Johanne.Lebrun@USherbooke.ca

daniel.niclot@univ-reims.fr

Texte reçu: 17 avril 2008

Accepté le: 18 avril 2008 